\title{
THE FAMILY OF ALL RECURSIVELY ENUMERABLE CLASSES OF FINITE SETS( $\left.{ }^{1}\right)$
}

\author{
BY \\ T. G. McLAUGHLIN
}

\begin{abstract}
We prove that if $P(x)$ is any first-order arithmetical predicate which enumerates the family Fin of all r.e. classes of finite sets, then $P(x)$ must reside in a level of the Kleene hierarchy at least as high as $\Pi_{3}^{0}-\Sigma_{3}^{0}$. (It is more easily established that some of the predicates $P(x)$ which enumerate Fin do lie in $\Pi_{3}^{0}-\Sigma_{3}^{0}$.)
\end{abstract}

1. Introduction. It has been remarked by C. E. M. Yates, in a footnote on p. 338 of [7], that the family Fin of all recursively enumerable classes of finite subsets of $N(N=$ the set of all natural numbers $)$ is not a recursively enumerable family. Since the family Fin is involved in many of the constructions which occur in recursion theory, the exact location of its "level of enumerability" in the Kleene hierarchy seems to us to be a natural and perhaps even useful undertaking. It is trivial to show that Fin can be enumerated by a $\Sigma_{4}^{0}$ predicate; thus, since Yates' remark amounts to the assertion that Fin cannot be enumerated by a $\Sigma_{1}^{0}$ predicate, it remains to consider the levels $\Sigma_{2}^{0}$ and $\Sigma_{3}^{0}$. We settle the matter by means of Theorem 2 in $\S 3$, which implies: Fin cannot be enumerated by a $\Sigma_{3}^{0}$ predicate. On the other hand, it follows from a theorem of Rogers [6, p. 326, Theorem XV] that the index set $G\left(\right.$ Fin), as defined below, is a complete $\Pi_{3}^{0}$ set of numbers; in $\S 2$ we shall prove a general assertion about index sets corresponding to families, from which Rogers' theorem is readily derivable.

In the remainder of this introductory section, we set forth the notational and terminological conventions which are to be in force in $\S \S 2,3 . N$ denotes the set $\{0,1,2, \ldots\}$ of natural numbers. Lower case Greek letters other than $\delta$ and $\rho$ denote either subsets of $N$ or partial number-theoretic functions (i.e., functions from a domain $\beta$ into $N$, where $\beta \subseteq N \times \cdots \times N$ ( $k$ times) for some $k \geqq 1$ ); context will determine, in any particular case, whether a subset or a function is meant. For each $k \geqq 1$, let the predicate $T_{k}\left(z, x_{1}, \ldots, x_{k}, y\right)$ be defined as in [4, §57]. Then with

Received by the editors February 26, 1968 and, in revised form, June 18, 1970.

AMS 1969 subject classifications. Primary 0270, 0277; Secondary 0280.

Key words and phrases. Recursively enumerable class of finite sets, Kleene hierarchy, $\Sigma_{n}^{0}$-enumerability, $\Sigma_{n}^{0}$-productivity.

(1) The main content of this paper was developed by the author while visiting at Cornell University during the academic year 1966-67. Preparation for publication supported under NSF Grant GP-7421 at the University of Illinois. 
notation as in $[4, \S 63]$, we obtain a recursive enumeration $\left\{\varphi_{e}^{k}\right\}_{e=0}^{\infty}$ of the $k$-place partial recursive functions by means of the definition:

$$
\varphi_{e}^{k}\left(x_{1}, \ldots, x_{k}\right) \simeq U\left(\mu y T_{k}\left(e, x_{1}, \ldots, x_{k}, y\right)\right) .
$$

It is easily seen from the definition of $T_{k}$ that $\varphi_{0}^{k}=\varnothing$ for all $k$. If $\psi$ is a partial number-theoretic function, we denote by $\delta \psi$ the domain of $\psi$ and by $\rho \psi$ the range of $\psi$. If $\psi$ is a partial number-theoretic function of $k$ variables, where $k \geqq 2$, and if $a_{1}, \ldots, a_{k-1}$ are constants, then by $\delta \psi\left(a_{1}, \ldots, a_{k-1}, x\right)$ we mean

$$
\left\{x \mid \psi\left(a_{1}, \ldots, a_{k-1}, x\right) \text { is defined }\right\} .
$$

For each $e$, we denote by $W_{e}$ the set $\delta \varphi_{e}^{1}$. Thus in our notation $W_{e}=$ the $e$ th recursively enumerable set; we abbreviate "recursively enumerable" as "r.e.". For each $k \leqq 1$, we denote by $\Sigma_{k}^{0}$ the class of predicates $P(x)$ such that, for some $e$, $P(x) \Leftrightarrow\left(\exists x_{1}\right)\left(\forall x_{2}\right) \cdots\left(Q x_{k}\right) T_{k}^{*}\left(e, x, x_{1}, \ldots, x_{k}\right)$, where the quantifiers alternate between existential and universal and $T_{k}^{*}$ is either $T_{k}$ or $\neg T_{k}$ according as $k$ is odd or even ( $Q=\exists$ if $k$ is odd; $Q=\forall$ if $k$ is even). We denote by $\Pi_{k}^{0}$ the class $\{R(x) \mid$ for some $P(x) \in \Sigma_{k}^{0}$ we have $\left.P(x) \Leftrightarrow \neg R(x)\right\}$; i.e., the predicates in $\Pi_{k}^{0}$ are the negations of those in $\Sigma_{k}^{0}$. It is well known that the class $\Sigma_{k}^{0}$ includes all predicates expressible in the form $\left(\exists x_{1}\right)\left(\forall x_{2}\right) \cdots\left(Q x_{k}\right) R\left(x, x_{1}, \ldots, x_{k}\right)$ where $R\left(x, x_{1}, \ldots, x_{k}\right)$ is a recursive predicate. We shall denote by $\Sigma[k ; e]$ the set

$$
\left\{x \mid\left(\exists x_{1}\right)\left(\forall x_{2}\right) \cdots\left(Q x_{k}\right) T_{k}^{*}\left(e, x, x_{1}, \ldots, x_{k}\right)\right\}, \quad k \geqq 1 .
$$

(Note that $\Sigma[1 ; e]=W_{e}$ for all e.) A predicate $P(x)$ in $\Sigma_{k}^{0}\left(\right.$ in $\Pi_{k}^{0}$ ) is called complete $\Leftrightarrow$ [for every predicate $S(x)$ in $\Sigma_{k}^{0}$ (in $\Pi_{k}^{0}$ ) there exists a recursive function $\psi_{S}$ such that $\left.S(x) \Leftrightarrow P\left(\psi_{s}(x)\right)\right]$. Let $\beta=\{x \mid P(x)$ is true $\}$; then $\beta$ is complete (for $\Sigma_{k}^{0}$ or $\left.\Pi_{k}^{0}\right) \Leftrightarrow P(x)$ is complete (for $\Sigma_{k}^{0}$ or $\Pi_{k}^{0}$, correspondingly). For each $k \geqq 2$, let $\pi_{k}$ denote a fixed recursive " $k$-tupling" function: $\pi_{k}$ maps $N \times \cdots \times N$ ( $k$ times) oneone onto $N$. Let $\tau_{k}^{1}, \ldots, \tau_{k}^{k}$ be the (recursive) "converses" of $\pi_{k}$, i.e.,

$$
\pi_{k}\left(\tau_{k}^{1}(x), \ldots, \tau_{k}^{k}(x)\right)=x,
$$

for all $x$. Thus, for each $k \geqq 2, \pi_{k}\left(x_{1}, \ldots, x_{k}\right)$ is a "Gödel number" of the ordered $k$-tuple $\left\langle x_{1}, \ldots, x_{k}\right\rangle=\left\langle\tau_{k}^{1}\left(\pi_{k}\left(x_{1}, \ldots, x_{k}\right)\right), \ldots, \tau_{k}^{k}\left(\pi_{k}\left(x_{1}, \ldots, x_{k}\right)\right)\right\rangle$. For each $k \geqq 1$, fix a recursive enumeration $\mathscr{E}_{k}$ of the set $\left\{\left\langle e, x_{1}, \ldots, x_{k}, y\right\rangle \mid \varphi_{e}^{k}\left(x_{1}, \ldots, x_{k}\right)=y\right\}$; then denote by $\varphi_{e}^{k, s}$ the set $\left\{\left\langle x_{1}, \ldots, x_{k}, y\right\rangle \mid(\exists t)_{t \leqq s}\left(\mathscr{E}_{k}(t)=\left\langle e, x_{1}, \ldots, x_{k}, y\right\rangle\right)\right\}$. We denote by $p_{n}$ the $n$th prime number in order of magnitude (starting the indexing at $\left.p_{0}=2\right)$ and by $P_{n}$ the set $\left\{p_{n}^{r} \mid r \in N\right\}$; then $(m)_{n}$ denotes, as usual, the power to which $p_{n}$ divides $m$ (with $(0)_{n}=0$ for all $n$ ). In addition to r.e. sets, we wish to consider r.e. classes of r.e. sets and also families of r.e. classes of r.e. sets. By an r.e. class we mean a class $K$ of r.e. sets such that, for some $e, K=\left\{W_{x} \mid x \in W_{e}\right\}$. If $K=\left\{W_{x} \mid x \in W_{e}\right\}$, we refer to $e$ as an index of $K$; and we define, for each $e, W_{e}^{C}$ $=\left\{W_{x} \mid x \in W_{e}\right\}$. The letters $K$ and $L$ shall henceforth be used to denote classes (whether r.e. or not) of r.e. sets. By an r.e. family we mean a family $\mathscr{F}$ of r.e. classes such that, for some $e, \mathscr{F}=\left\{W_{x}^{C} \mid x \in W_{e}\right\}$. If $\mathscr{F}=\left\{W_{x}^{C} \mid x \in W_{e}\right\}$, we refer to 
$e$ as an r.e. index of $\mathscr{F}$; and we define, for each $e, W_{e}^{F}=\left\{W_{x}^{C} \mid x \in W_{e}\right\}$. More generally, by a $\Sigma_{k}^{0}$ family $(k \geqq 1)$ we mean a family $\mathscr{F}$ of r.e. classes such that, for some $e, \mathscr{F}=\left\{W_{x}^{C} \mid x \in \Sigma[k ; e]\right\}$. If $\mathscr{F}=\left\{W_{x}^{C} \mid x \in \Sigma[k ; e]\right\}$, we refer to the pair $\langle k, e\rangle$ as a $\Sigma_{k}^{0}$ index of $\mathscr{F}$; and we define, for each $e, \Sigma[k ; e]^{F}=\left\{W_{x}^{C} \mid x \in \Sigma[k ; e]\right\}$. Capital script letter such as $\mathscr{F}$ and $\mathscr{G}$ are used to denote families (whether r.e. or not) of r.e. classes. If $\mathscr{F}$ is any family of r.e. classes, the index set, $G(\mathscr{F})$, corresponding to $\mathscr{F}$ is defined by $G(\mathscr{F})=\left\{e \mid W_{e}^{C} \in \mathscr{F}\right\}$. It is easily seen that there exists a recursive function $\zeta_{0}$ such that for all $e$ we have

$$
W_{k}^{C} \in W_{e}^{F} \Leftrightarrow(\exists j)(\forall h)\left[W_{h} \in W_{k}^{C} \Leftrightarrow(\exists l)\left(W_{h}=\delta \varphi_{\zeta_{0}(e)}^{3}(j, l, x)\right)\right]
$$

Conversely, there is a recursive function $\zeta_{1}$ such that for all $e$ we have

$$
W_{k}^{C} \in W_{\zeta_{1}(e)}^{F} \Leftrightarrow(\exists j)(\forall h)\left[W_{h} \in W_{k}^{C} \Leftrightarrow(\exists l)\left(W_{h}=\delta \varphi_{e}^{3}(j, l, x)\right)\right] .
$$

Thus, three-place partial recursive functions are effectively interchangeable with collections $W_{e}^{F}$ as descriptions of r.e. families. A three-place partial recursive function $\varphi_{e}^{3}$, regarded as a description of an r.e. family $\mathscr{F}$, shall be termed an enumeration of $\mathscr{F}$; and we term $\varphi_{e}^{3}$ a row-disjoint enumeration (of whatever family it enumerates) if the following condition is satisfied:

$$
(\forall j)(\forall m)(\forall n)\left[m \neq n \Rightarrow \delta \varphi_{e}^{3}(j, m, x) \cap \delta \varphi_{e}^{3}(j, n, x)=\varnothing\right] .
$$

It will prove convenient in $\$ 3$ to work with the class of special retracing functions, i.e., those partial recursive functions which retrace at least one infinite subset of $N$ and possess properties (3) and (4) of [2, p. 81]; we shall here add the requirement that a special retracing function have only finitely many fixed points. Some use will be made of the notation $\gamma^{*}(x)$ ( $\gamma$ an arbitrary one-place partial number-theoretic function); the meaning of this notation is that prescribed in [2, p. 81]. For some of the basic properties of special retracing functions and of the mapping $\gamma \rightarrow \gamma^{*}$, the reader may consult [1], [2], and [7]. For any partial recursive function $\gamma$ such that $x \in \delta \gamma \Rightarrow \gamma(x) \leqq x$, we denote by $K_{\gamma}$ the disjoint r.e. class $\left\{\left\{n \mid n \in \delta \gamma \& \gamma^{*}(n)\right.\right.$ $=k\} \mid k \in N\}$; and we denote by $\boldsymbol{F} \boldsymbol{R}$ the family $\left\{K_{\gamma} \mid \gamma\right.$ is a finite-to-one special retracing function\}. Finally, we shall extend the notion of productive class (see [3]) in a natural way to the context of families of r.e. classes: for a given $k \geqq 1$, a family $\mathscr{F}$ of r.e. classes is $\Sigma_{k}^{0}$-productive $\Leftrightarrow$ there exists a partial recursive function $\psi$ such that

$$
(\forall e)\left[\Sigma[k ; e]^{F} \subseteq \mathscr{F} \Rightarrow\left(e \in \delta \psi \& W_{\psi(e)}^{C} \in \mathscr{F}-\Sigma[k ; e]^{F}\right)\right]
$$

such a function $\psi$ is termed a $\Sigma_{k}^{0}$-productive function for $\mathscr{F}$. If $\psi$ is a $\Sigma_{k}^{0}$-productive function for $\mathscr{F}$, we denote by $\{\mathscr{F} ; k ; \psi\}$ the family $\left\{W_{\psi(e)}^{C} \mid \Sigma[k ; e]^{F} \subseteq \mathscr{F}\right\}$ (which in the language of [3] would be called the $\Sigma_{k}^{0}$-productive center of $\mathscr{F}$ with respect to $\psi)$. 


\section{Classification of index sets corresponding to families.}

THEOREM 1. (i) If $\beta$ is complete at level $\Pi_{k}^{0}$, then $\left\{e \mid W_{e} \subseteq \beta\right\}$ is also complete at level $\Pi_{k}^{0}$.

(ii) If $\beta$ is complete at level $\Sigma_{k}^{0}$, then $\left\{e \mid W_{e} \subseteq \beta\right\}$ is complete at level $\Pi_{k+1}^{0}$.

Proof. Routine manipulations (as in $[6, \S 14.3]$ ) show that the predicate $W_{x} \subseteq \beta$ is in $\Pi_{k}^{0}$ in case (i) and in $\Pi_{k+1}^{0}$ in case (ii). Assume $\beta$ to be $\Sigma_{k}^{0}$ complete, and let $P(x)$ be any $\Pi_{k+1}^{0}$ predicate. Express $P(x)$ in the form $(\forall y) Q(x, y)$, where $Q(x, y)$ is a $\Sigma_{k}^{0}$ predicate of $x$ and $y$. Since $\beta$ is complete for $\Sigma_{k}^{0}$ predicates, there is a recursive function $\psi$ of two variables such that $(\forall y) Q(x, y) \Leftrightarrow(\forall y)[\psi(x, y) \in \beta]$. So let $f$ be a recursive function such that $(\forall x)\left[W_{f(x)}=\rho \psi(x, y)\right]$. Then $P(x) \Leftrightarrow W_{f(x)} \subseteq \beta$, and (ii) is proved. The proof of (i) is similar.

Corollary. G(Fin) is $\Pi_{3}^{0}$ complete.

Proof. Let $\beta=\left\{x \mid W_{x}\right.$ is finite $\}$. Then $\beta$ is $\Sigma_{2}^{0}$ complete, as is shown in [3]. Hence, by Theorem 1, $\left\{e \mid W_{e} \subseteq \beta\right\}$ is $\Pi_{3}^{0}$ complete. But obviously $\left\{e \mid W_{e} \subseteq \beta\right\}=G($ Fin). I

The above formulation of Theorem 1 was noted by Carl Jockusch, after its proof had been applied by the author to some special cases.

It is perhaps worth remarking that the index set corresponding to a recursively enumerable family of r.e. classes can reside at any of the following levels of the arithmetical hierarchy: $\Sigma_{1}^{0} \cap \Pi_{1}^{0}$ (i.e., recursive), complete $\Sigma_{1}^{0}$, complete $\Pi_{1}^{0}$, complete $\Sigma_{2}^{0}$, complete $\Pi_{2}^{0}$, complete $\Sigma_{3}^{0}$, complete $\Pi_{3}^{0}$, complete $\Sigma_{4}^{0}$, complete $\Pi_{4}^{0}$, complete $\Sigma_{5}^{0}$. Thus the above corollary provides no clue as to the possibility of $\Sigma_{k}^{0}$-enumerability of Fin for $k \leqq 3$.

3. $\Sigma_{3}^{0}$-productivity of Fin. We define Fin* $=$ the family of all disjoint r.e. classes of nonempty finite sets (the empty class not excluded), Fin ${ }_{\varnothing}^{*}=$ the family of all disjoint r.e. classes of finite sets (the empty class not excluded), Fin*o $=\left\{K \mid K \in\right.$ Fin $^{*} \& K$ is infinite $\}$, and $\operatorname{Fin}_{\varnothing}^{* \infty}=\left\{K \mid K \in\right.$ Fin $_{\varnothing}^{*} \& K$ is infinite $\}$. It is easy to see that $\boldsymbol{F R} \subseteq$ Fin $^{* \infty}$ and Fin ${ }^{* \infty} \nsubseteq \boldsymbol{F R}$; and in fact, by making minor changes in [5, proof of Theorem 3], one can produce much narrower families than Fin*o which properly include $\boldsymbol{F} \boldsymbol{R}$. Our first two lemmas serve as technical lubrication for the proof of Lemma $C$.

LEMMA A. There exists a recursive function $\xi$ such that

(1) $W_{e}^{F} \subseteq$ Fin $\Rightarrow \varphi_{\xi(e)}^{3}$ is a row-disjoint enumeration of a subfamily of Fin*, and

(2) $W_{f}^{C} \in W_{e}^{\boldsymbol{F}} \cap \boldsymbol{F R} \Rightarrow$ the family enumerated by $\varphi_{\xi(e)}^{3}$ contains the class $W_{f}^{C}$.

Proof. Let $\zeta$ be a recursive function such that $(\forall e)\left[\varphi_{\zeta(e)}^{3}\right.$ is an enumeration of $\left.W_{e}^{F}\right]$. We shall apply to each index $e$ a suitable "disjointification" of the rows of the enumeration $\varphi_{\zeta(e)}^{3}$; our procedure is uniform in $e$ (and results, in general, in some alteration of the given family $W_{e}^{F}$ ). In order to "disjointify" rows in a way appropriate to proving part (2) of the lemma, we shall treat the functions $\varphi_{n}^{1}$ as candidates for special retracing functions $\gamma$ whose associated classes $K_{y}$ we strive to locate among the rows of the enumeration $\varphi_{\zeta(e)}^{3}$. In detail, we proceed as follows. 
Stage $s$. Let $(s)_{0}=a,(s)_{1}=b,(s)_{2}=c,(s)_{3}=d$. We direct our attention to $\varphi_{a}^{1}$, to $\varphi_{\zeta(e)}^{3}(b, c, x)$, and to the numbers (if any) which $\varphi_{a}^{1}$ retraces to a fixed point in a minimum of $d$ applications. Specifically, consider $\varphi_{\zeta(e)}^{3, s}(b, c, x)$ and $\varphi_{a}^{1, s}$. If $\varphi_{a}^{1, s}$ does not have a nonempty subset of the form

$$
\left\{\left\langle y, \varphi_{a}^{1, s}(y)\right\rangle,\left\langle\varphi_{a}^{1, s}(y), \varphi_{a}^{1, s}\left(\varphi_{a}^{1, s}(y)\right)\right\rangle, \ldots,\left\langle\left[\varphi_{a}^{1, s}\right]^{d}(y),\left[\varphi_{a}^{1, s}\right]^{d+1}(y)\right\rangle\right\}
$$

where (i) $y>\varphi_{a}^{1, s}(y)>\cdots>\left[\varphi_{a}^{1, s}\right]^{d}(y)$, (ii) $\left[\varphi_{a}^{1, s}\right]^{d}(y)=\left[\varphi_{a}^{1, s}\right]^{d+1}(y)$, and (iii) $y \in \delta \varphi_{\zeta(e)}^{3 . s}(b, c, x)$, then we set $\kappa_{e}^{(s)}=\kappa_{e}^{(s-1)}\left(\kappa_{e}^{(s)}=\varnothing\right.$ if $\left.s=0\right)$ and proceed to stage $s+1$. Suppose, on the other hand, that such a subset of $\varphi_{a}^{1, s}$ does exist. Two cases arise.

Case 1. There is a number $c^{\prime} \neq c$ such that for some $y$ and some $s^{\prime}<s$ we have (i') $\varphi_{a}^{1, s^{\prime}}$ contains a subset of the form

$$
\left\{\left\langle y, \varphi_{a}^{1, s^{\prime}}(y)\right\rangle,\left\langle\varphi_{a}^{1, s^{\prime}}(y), \varphi_{a}^{1, s^{\prime}}\left(\varphi_{a}^{1, s^{\prime}}(y)\right)\right\rangle, \ldots,\left\langle\left[\varphi_{a}^{1, s^{\prime}}\right]^{d}(y),\left[\varphi_{a}^{1, s^{\prime}}\right]^{d+1}(y)\right\rangle\right\},
$$

where (ii') $y>\varphi_{a}^{1, s^{\prime}}(y)>\cdots>\left[\varphi_{a}^{1, s^{\prime}}\right]^{d}(y), \quad\left(\mathrm{iii}^{\prime}\right) \quad\left[\varphi_{a}^{1, s^{\prime}}\right]^{d}(y)=\left[\varphi_{a}^{1, s^{\prime}}\right]^{d+1}(y), \quad\left(\mathrm{iv}^{\prime}\right)$ $y \in \delta \varphi_{\zeta(e)}^{3, s^{\prime}}\left(b, c^{\prime}, x\right)$, and $\left(\mathrm{v}^{\prime}\right)\left\langle\pi_{2}(a, b), d, y, 0\right\rangle \in \kappa_{e}^{\left(s^{\prime}\right)}$.

In this case we set $\kappa_{e}^{(s)}=\kappa_{e}^{(s-1)}$ and proceed to stage $s+1$.

Case 2. Case 1 does not hold. Let $x_{0}, \ldots, x_{l}$ be all those members $m$ of $\delta \varphi_{\zeta(e)}^{3, s}(b, c, x)$ for which there is a subset

$$
\left\{\left\langle m, \varphi_{a}^{1, s}(m)\right\rangle,\left\langle\varphi_{a}^{1, s}(m), \varphi_{a}^{1, s}\left(\varphi_{a}^{1, s}(m)\right)\right\rangle, \ldots,\left\langle\left[\varphi_{a}^{1, s}\right]^{d}(m),\left[\varphi_{a}^{1, s}\right]^{d+1}(m)\right\rangle\right\}
$$

of $\varphi_{a}^{1, s}$ with $m>\varphi_{a}^{1, s}(m)>\cdots>\left[\varphi_{a}^{1, s}\right]^{d}(m)$ and $\left[\varphi_{a}^{1, s}\right]^{d+1}(m)=\left[\varphi_{a}^{1, s}\right]^{d}(m)$.

Set $\left.\kappa_{e}^{(s)}=\kappa_{e}^{(s-1)}\right) \cup\left\{\left\langle\pi_{2}(a, b), d, m, 0\right\rangle \mid m \in\left\{x_{0}, \ldots, x_{l}\right\}\right\}$; then proceed to stage $s+1$.

That completes our description of stage $s$ in the construction of $\kappa_{e}$, where $\kappa_{e}=\bigcup_{s} \kappa_{e}^{(s)}$; we must verify that $\kappa_{e}$ is a partial recursive function suitable for use as $\varphi_{\xi(e)}^{3}$. Certainly $\kappa_{e}$ is a function, since the only number which ever occurs as a value $\tau_{4}^{4}(n), n \in \kappa_{e}$, is 0 . But it is also plain that $\kappa_{e}$ is an r.e. set of quadruples obtained in a uniform effective way from $e$. Thus, $\kappa_{e}$ is a three-place partial recursive function which we may represent as $\varphi_{\xi(e)}^{3}, \xi$ a recursive function of $e$. We next assume that $W_{e}^{\boldsymbol{F}} \subseteq$ Fin, and show that $\kappa_{e}$ provides a row-disjoint enumeration of a subfamily of Fin ${ }_{\varnothing}^{*}$. Fix a number $n$, and consider the $n$th row enumerated by $\kappa_{e}$. The $m$ th set in this row is given by $\delta \kappa_{e}(n, m, x)$. Now, it is clear from the construction of $\kappa_{e}$ that if $\delta \kappa_{e}(n, m, x) \neq \varnothing$ then there exists a number $c_{0}$ such that $\delta \kappa_{e}(n, m, x)$ consists entirely of elements of $\delta \varphi_{\zeta(e)}^{3}\left(\tau_{2}^{2}(n), c_{0}, x\right)$ which are retraced by $\varphi_{\tau_{2}^{1}(n)}^{1}$ to a fixed point in exactly $m$ steps. But $\delta \varphi_{\zeta(e)}^{3}\left(\tau_{2}^{2}(n), c_{0}, x\right)$ is finite since $W_{e}^{F} \subseteq$ Fin; hence $\delta \kappa_{e}(n, m, x)$ is also finite. That $\kappa_{e}$ is a row-disjoint enumeration of the family which it enumerates is clear from the fact, just cited, that all elements of $\delta \kappa_{e}(n, m, x)$ have height exactly $=m$ under iterated application of $\varphi_{\tau_{2}^{1}(n)}^{1}$.

It now remains to show that if $W_{f}^{C} \in W_{e}^{F} \cap \boldsymbol{F R}$, then some row of the enumeration given by $\kappa_{e}$ precisely covers the membership of $W_{f}^{C}$. But if $W_{f}^{C} \in W_{e}^{F} \cap \boldsymbol{F R}$, 
then there is a special, finite-to-one retracing function $\varphi_{a}^{1}$ such that $W_{f}^{C}=K_{\varphi_{a}^{1}}$; moreover, some row $\varphi_{\zeta(e)}^{3}(b, x, y)$ in the enumeration $\varphi_{\zeta(e)}^{3}$ of $W_{e}^{F}$ enumerates precisely the class $W_{f}^{C}$. It is clear from the construction of $\kappa_{e}$ that these two facts imply enumeration of $W_{f}^{C}$ by the row $\kappa_{e}\left(\pi_{2}(a, b), x, y\right)$.

Lemma B. There exists a recursive function $\mu$ such that $\left\{\Sigma[3 ; e]^{F} \subseteq\right.$ Fin $\& x$ $\in \Sigma[3 ; e]\} \Rightarrow\left\{\varphi_{\mu(x)}^{3}\right.$ is a row-disjoint enumeration of a subfamily $\mathscr{G}$ of Fin such $^{*}$ that $\left(W_{x}^{C} \in \boldsymbol{F R} \Rightarrow W_{x}^{C}\right.$ is enumerated by some row of the enumeration $\left.\left.\varphi_{\mu(x)}^{3}\right)\right\}$. (More generally, and in virtue of the same proof, replace $\Sigma[3 ; e]$ by an arbitrary set $\alpha$ of natural numbers, and replace $\Sigma[3 ; e]^{F}$ by the family $\mathscr{F}=\left\{W_{x}^{C} \mid x \in \alpha\right\}$.)

Proof. Let $\xi$ be as in Lemma $\mathrm{A}$, and let $\beta$ be a recursive function such that $W_{\beta(x)}=\{x\}$ holds for all $x$. Then it is easily deduced from the statement of Lemma A that the function $\mu$ defined by $\mu(x)=\xi(\beta(x))$ has the required property.

LEMMA C. There exists a recursive function $\psi$ such that $\psi$ is $\Sigma_{3}^{0}$-productive for Fin and $\{$ Fin $; 3 ; \psi\} \subseteq \boldsymbol{F R}$.

Proof. We shall make use of a collection $\left\{\Lambda_{\langle a, b, c\rangle}\right\}$ of "markers", one for each ordered triple $\langle a, b, c\rangle$ of natural numbers. We impose an ordering on these markers by the rule:

$$
\Lambda_{\left\langle a_{1}, b_{1}, c_{1}\right\rangle} \prec \Lambda_{\left\langle a_{2}, b_{2}, c_{2}\right\rangle} \Leftrightarrow \pi_{3}\left(a_{1}, b_{1}, c_{1}\right)<\pi_{3}\left(a_{2}, b_{2}, c_{2}\right) .
$$

Let $\mu$ be a recursive function as in Lemma B. The rough idea of our procedure is this: We assume a $\Sigma_{3}^{0}$ predicate $(\exists w)(\forall z)(\exists y) R(x, w, z, y)$ to be fixed, and we consider a particular number $a . \Lambda_{\langle a, b, c\rangle}$ is used to keep track, insofar as possible, of events in the $b$ th row of the enumeration $\varphi_{\mu(a)}^{3}$; we move $\Lambda_{\langle a, b, c\rangle}$ so as to contribute to the production of a suitable class in $\boldsymbol{F} \boldsymbol{R}$. The movement of $\Lambda_{\langle a, b, c\rangle}$ is restricted by the condition that we may move $\Lambda_{\langle a, b, c\rangle}$ autonomously for the $n$th time only after having verified that $(\forall z \leqq n)(\exists y) R(a, c, z, y)$; an "autonomous" move of $\Lambda_{\langle a, b, c\rangle}$ is one which is not occasioned simply by the fact that some marker $\Lambda_{\langle d, e, f\rangle}$ is moved where $\pi_{3}(d, e, f)<\pi_{3}(a, b, c)$. We proceed now to the details of the construction. For notational convenience, we shall abbreviate $\left\langle\tau_{3}^{1}(k), \tau_{3}^{2}(k), \tau_{3}^{3}(k)\right\rangle$ to $\langle k\rangle^{-1}$, for all $k$.

Stage 0 . Attach $\Lambda_{\langle 0\rangle}{ }^{-1}$ to 0 , set $\alpha^{(0)}=\{\langle 0,0\rangle\}$, and proceed to stage 1 .

Stage $s, s>0$. We assume it to have been arranged that, at the conclusion of stage $s-1$, exactly the members of the initial segment $\left.\Lambda_{\langle 0\rangle}^{-1}, \Lambda_{\langle 1\rangle}{ }^{-1}, \ldots, \Lambda_{\langle s-1\rangle}\right\rangle^{-1}$ of markers are attached to numbers (that this assumption is allowable will be manifest from our description of the remainder of the construction); and we shall denote by $\lambda_{j}^{s-1}$ the number to which $\left.\Lambda_{\langle j\rangle}\right\rangle^{-1}$ is attached at the end of stage $s-1$, $0 \leqq j \leqq s-1$. We shall assume further that a number $e$ has been fixed, and deal throughout the remainder of our description of stage $s$ with the fixed predicate $(\exists w)(\forall z)(\exists y) T_{3}(e, x, w, z, y)$; once the procedure has been described in full, it will be obvious that it is uniform in $e$. Now, we wish to move in a suitable way the least 
marker, if any, whose position is currently insecure; so we must explain what we mean in saying that a marker position is currently (i.e., at stage $s$ ) insecure. In our definition of insecurity, marker positions will be referred to as being associated with certain members of certain rows of certain recursive enumerations of classes; how this association comes about will be clear from the main part of the construction, following the definition. We remind the reader that $\mu$ is as in Lemma B.

Definition. Consider $\lambda_{j}^{s-1}$, where $0<j \leqq s-1$; and let $\pi_{3}^{-1}(j)=\langle a, b, c\rangle . \lambda_{j}^{s-1}$ is insecure if and only if the following conditions are satisfied:

(1) $(\forall z \leqq r+1)(\exists y \leqq s) T_{3}(e, a, c, z, y)$, where $r=$ the number of previous autonomous moves (i.e., autonomous moves during stages $t<s$ ) of $\Lambda_{\langle j\rangle}^{-1}$; and

(2) $\lambda_{j}^{s-1}$ is currently associated with a term $\varphi_{\mu(a)}^{3}\left(b, t_{j}, x\right)$ such that

$$
(\exists l \leqq j)(\exists n)\left[n \in \delta \varphi_{\mu(a)}^{3, s}\left(b, t_{j}, x\right) \&\left[\left(l<j \& \lambda_{l}^{s-1} \geqq n\right) \vee\left(l=j \& \lambda_{l}^{s-1} \leqq n\right)\right]\right] .
$$

The procedure now splits into cases, according as there does or does not exist an insecure marker position.

Case I. For all $j$ such that $0 \leqq j \leqq s-1, \lambda_{j}^{s-1}$ is not insecure. In this case, our only concern is to attach $\Lambda_{\langle s\rangle}{ }^{-1}$ in a suitable way. Let $\pi_{3}^{-1}(s)=\left\langle a_{1}, b_{1}, c_{1}\right\rangle$. Let $t_{s}$ be the smallest number $t$ with the following properties:

(i) $(\forall l \leqq s-1)\left(\forall n \in \delta \varphi_{\mu\left(a_{1}\right)}^{3, s}\left(b_{1},(t)_{0}, x\right)\right)\left(n>\lambda_{l}^{s-1}\right)$,

(ii) $\left(\forall n \in \delta \varphi_{\mu\left(a_{1}\right)}^{3, s}\left(b_{1},(t)_{0}, x\right)\right)\left(n<(t)_{1}\right)$, and

(iii) $(t)_{1}>\max \left\{m \mid(\exists q \leqq s-1)(\exists u \leqq s-1)\left(m=\lambda_{u}^{q}\right)\right\}$.

(We remark that the function which maps $s$ to

$$
\max \left\{m \mid(\exists q \leqq s-1)(\exists u \leqq s-1)\left(m=\lambda_{u}^{q}\right)\right\}
$$

is recursive; this will be evident once our description of stage $s$ is complete.) We attach $\left.\Lambda_{\langle s\rangle}\right\rangle^{-1}$ to $\left(t_{s}\right)_{1}$, associate $\left(t_{s}\right)_{1}$ with the term $\varphi_{\mu\left(a_{1}\right)}^{3}\left(b_{1},\left(t_{s}\right)_{0}, x\right)$, set $\alpha^{(s)}$ $=\alpha^{(s-1)} \cup\left\{\left\langle\left(t_{s}\right)_{1}, \lambda_{s-1}^{s-1}\right\rangle\right\}$, and proceed to stage $s+1$.

Case II. There exists a number $j, 0<j \leqq s-1$, such that $\lambda_{j}^{s-1}$ is insecure. Let $j_{0}=$ the least such $j$, and let $\pi_{3}^{-1}\left(j_{0}\right)=\left\langle a_{2}, b_{2}, c_{2}\right\rangle$. We take $t_{j_{0}}$ to be the smallest number $t$ with the following properties:

(i') $\left(\forall l<j_{0}\right)\left(\forall n \in \delta \varphi_{\mu\left(a_{2}\right)}^{3, s}\left(b_{2},(t)_{0}, x\right)\right)\left(n>\lambda_{l}^{s-1}\right)$,

(ii') $\left(\forall n \in \delta \varphi_{\mu\left(a_{2}\right)}^{3, s}\left(b_{2},(t)_{0}, x\right)\right)\left(n<(t)_{1}\right)$, and

(iii') $(t)_{1}>\max \left\{m \mid(\exists q \leqq s-1)(\exists u \leqq s-1)\left(m=\lambda_{u}^{q}\right)\right\}$.

If $\left(\exists l<j_{0}\right)\left(\lambda_{l}^{s-1} \geqq n\right.$ for some $\left.n \in \delta \varphi_{\mu\left(a_{2}\right)}^{3, s}\left(b_{2}, z_{0}, x\right)\right)$, where $\varphi_{\mu\left(a_{2}\right)}^{3}\left(b_{2}, z_{0}, x\right)$ is the term with which $\lambda_{j_{0}}^{s-1}$ was associated at the beginning of stage $s$, then we remove $\Lambda_{\left\langle j_{0}\right\rangle^{-1}}$ from $\lambda_{j_{0}}^{s-1}$, reattach $\Lambda_{\left\langle j_{0}\right\rangle^{-1}}$ to $\left(t_{j_{0}}\right)_{1}$, associate $\left(t_{j_{0}}\right)_{1}$ with the term $\delta_{\mu\left(a_{2}\right)}^{3}\left(b_{2},\left(t_{j_{0}}\right)_{0}, x\right)$, and remove $\Lambda_{\langle k\rangle}{ }^{-1}$ from its present position $\lambda_{k}^{s-1}$ for all $k$ such that $j_{0}<k \leqq s-1$. If no such $l$ exists, then it must be the case that $\lambda_{j_{0}}^{s-1} \leqq n$ for some $n \in \delta \varphi_{\mu\left(a_{2}\right)}^{3 . s}\left(b_{2}, z_{0}, x\right)$. In this event, we move $\left.\Lambda_{\left\langle j_{0}\right\rangle}\right\rangle^{-1}$ as before, but associate its new position $\left(t_{j_{0}}\right)_{1}$ with $\varphi_{\mu\left(a_{2}\right)}^{3}\left(b_{2}, z_{0}, x\right)$ (i.e., with the same term to which $\lambda_{j_{0}}^{s-1}$ was associated). The movement just imposed on $\Lambda_{\left\langle j_{0}\right\rangle}{ }^{-1}$ counts as an autonomous move. 
We shall reattach $\Lambda_{\left\langle j_{0}+1\right\rangle^{-1}}, \ldots, \Lambda_{\langle s-1\rangle^{-1}}$ (and, also, shall newly attach $\left.\Lambda_{\langle s\rangle}\right\rangle^{-1}$ ); but their moves are not counted as autonomous.

Next we reattach $\Lambda_{\left\langle j_{0}+1\right\rangle}{ }^{-1}$ (unless $j_{0}=s-1$ ). Let $\pi_{3}^{-1}\left(j_{0}+1\right)=\left\langle a_{3}, b_{3}, c_{3}\right\rangle$; and let $t_{j_{0}+1}$ be the smallest number $t$ with the following properties:

(i") $\left(\forall l<j_{0}\right)\left(\forall n \in \delta \varphi_{\mu\left(a_{3}\right)}^{3, s}\left(b_{3},(t)_{0}, x\right)\right)\left(n>\lambda_{l}^{s-1}\right) \&\left(\forall n \in \delta \varphi_{\mu\left(a_{3}\right)}^{3, s}\left(b_{3},(t)_{0}, x\right)\right)\left(n>\lambda_{j_{0}}^{s}\right.$ $\left.=\left(t_{j_{0}}\right)_{1}\right)$,

(ii") $\left(\forall n \in \delta \varphi_{\mu\left(a_{3}\right)}^{3, s}\left(b_{3},(t)_{0}, x\right)\right)\left(n<(t)_{1}\right)$, and

(iii") $(t)_{1}>\max \left\{m \mid(\exists q \leqq s-1)(\exists u \leqq s-1)\left[m=\lambda_{u}^{q} \vee m=\left(t_{j_{0}}\right)_{1}\right]\right\}$.

We attach $\Lambda_{\left\langle j_{0}+1\right\rangle}{ }^{-1}$ to $\left(t_{j_{0}+1}\right)_{1}$ and associate $\left(t_{j_{0}+1}\right)_{1}$ with the term

$$
\varphi_{\mu\left(a_{3}\right)}^{3}\left(b_{3},\left(t_{j_{0}+1}\right)_{0}, x\right) \text {. }
$$

(Our assumption here is that $j_{0}<s-1$.) In general, let us suppose that we have accomplished the reattachment of $\left.\Lambda_{\left\langle j_{0}+1\right\rangle}{ }^{-1}, \ldots, \Lambda_{\left\langle j_{0}+p\right\rangle}\right\rangle^{-1}, p \geqq 1, j_{0}+p+1<s-1$. Let us designate by $\lambda_{0}^{s}, \lambda_{1}^{s}, \ldots, \lambda_{j_{0}}^{s}, \lambda_{j_{0}+1}^{s}, \ldots, \lambda_{j_{0}+p}^{s}$ the numbers to which $\left.\left.\Lambda_{\langle 0\rangle}\right\rangle^{-1}, \Lambda_{\langle 1\rangle}-1, \ldots, \Lambda_{\left\langle j_{0}\right\rangle^{-1}}, \Lambda_{\left\langle j_{0}+1\right\rangle}\right\rangle^{-1}, \ldots, \Lambda_{\left\langle j_{0}+p\right\rangle^{-1}}$ are attached following this process of reattachment. Let $\pi_{3}^{-1}\left(j_{0}+p+1\right)=\left\langle a_{4}, b_{4}, c_{4}\right\rangle$; and let $t_{j_{0}+p+1}$ be the smallest number $t$ with the properties:

(i') $\left(\forall l \leqq j_{0}+p\right)\left(\forall n \in \delta \varphi_{\mu\left(a_{4}\right)}^{3, s}\left(b_{4},(t)_{0}, x\right)\right)\left(n>\lambda_{l}^{s}\right)$,

(ii") $\left(\forall n \in \delta \varphi_{\mu\left(a_{4}\right)}^{3, s}\left(b_{4},(t)_{0}, x\right)\right)\left(n<(t)_{1}\right)$, and

(iii"') $(t)_{1}>\max \left\{m \mid(\exists q \leqq s-1)(\exists u \leqq s-1)\left(m=\lambda_{u}^{q}\right) \vee\left(\exists u \leqq j_{0}+p\right)\left(m=\lambda_{u}^{s}\right)\right\}$.

We attach $\left.\Lambda_{\left\langle j_{0}+p+1\right\rangle}\right\rangle^{-1}$ to $\left(t_{j_{0}+p+1}\right)_{1}$ and associate $\left(t_{j_{0}+p+1}\right)_{1}$ with the term $\varphi_{\mu\left(a_{4}\right)}^{3}\left(b_{4},\left(t_{j_{0}+p+1}\right)_{0}, x\right)$. We then designate $\left(t_{j_{0}+p+1}\right)_{1}$ as $\lambda_{j_{0}+p+1}^{s}$.

This procedure continues until all of $\Lambda_{\left\langle j_{0}\right\rangle}{ }^{-1}, \ldots, \Lambda_{\langle s-1\rangle}{ }^{-1}$ are present in their new positions; it then remains only to attach $\Lambda_{\langle s\rangle}{ }^{-1}$. To attach $\Lambda_{\langle s\rangle^{-1}}$, we proceed (apart from extending $\alpha$ ) exactly as in Case I, but of course using the numbers $\lambda_{l}^{s}, l \leqq s-1$, in place of the "old" numbers $\lambda_{l}^{s-1}$. Finally, we make our extension of $\alpha$ as follows:

$$
\alpha^{(s)}=\alpha^{(s-1)} \cup\left\{\left\langle\lambda_{j_{0}}^{s}, \lambda_{j_{0}-1}^{s}\right\rangle,\left\langle\lambda_{j_{0}+1}^{s}, \lambda_{j_{0}}^{s}\right\rangle, \ldots,\left\langle\lambda_{s-1}^{s}, \lambda_{s-2}^{s}\right\rangle,\left\langle\lambda_{s}^{s}, \lambda_{s-1}^{s}\right\rangle\right\} .
$$

We then proceed to stage $s+1$.

That completes our description of stage $s$.

It remains to see that our construction has the desired effect. We shall first argue that if $\Sigma[3 ; e]^{F} \subseteq$ Fin then, for all $k, \lim _{s \rightarrow \infty} \lambda_{k}^{s}$ exists; i.e., all markers achieve final positions. Assume, then, that $\Sigma[e ; 3]^{F} \subseteq$ Fin. To begin with, it is obvious from the descriptions given of stages 0 and $s>0$ that $\Lambda_{\langle 0\rangle}{ }^{-1}$, after being attached to 0 at stage 0 , never moves; hence $\lambda_{0}=\lim _{s \rightarrow \infty} \lambda_{0}^{s}$ exists and $=0$. Now assume that $\lambda_{j}=\lim _{s \rightarrow \infty} \lambda_{j}^{s}$ exists for all $j \leqq k$, and consider $\Lambda_{\langle k+1\rangle}-1$. Let $t$ be a stage $\geqq k+1$ (so that $\lambda_{k+1}^{u}$ is defined for all $u \geqq t$ ) such that $\lambda_{j}^{t-1}=\lambda_{j}$ for all $j \leqq k$. Thus, $\Lambda_{\langle k+1\rangle^{-1}}$ can only move autonomously at stages $u \geqq t$. So it suffices to show that $\lambda_{k+1}^{u}$ cannot be insecure at more than finitely many stages $u \geqq t$. Now, if $\lambda_{k+1}^{u_{1}}$ is insecure at $u_{1} \geqq t$ (indeed, at any stage $u_{1}$ ), then, in particular,

$$
(\forall z \leqq r+1)\left(\exists y \leqq u_{1}+1\right) T_{3}(e, a, c, z, y),
$$


where $\langle a, b, c\rangle=\pi_{3}^{-1}(k+1)$ for some $b$ and where $r=$ the number of previous autonomous moves of $\Lambda_{\langle k+1\rangle}{ }^{-1}$ (note that $r \leqq$ the number of $s<u_{1}$ for which $\lambda_{k+1}^{s}$ is insecure). So, if there are infinitely many $u \geqq t$ for which $\lambda_{k+1}^{u}$ is insecure, then, since $\Lambda_{\langle k+1\rangle^{-1}}$ must move autonomously at all such $u$, we have that $(\forall z)(\exists y) T_{3}(e$, $a, c, z, y)$. Thus $(\exists w)(\forall z)(\exists y) T_{3}(e, a, w, z, y)$; so $a \in \Sigma[3 ; e]$. Hence, by Lemma $\mathrm{B}, \varphi_{\mu(a)}^{3}$ is a row-disjoint enumeration of a family of classes of finite sets. By the rowdisjointedness of $\varphi_{\mu(a)}^{3}$, finitely many moves of $\Lambda_{\langle k+1\rangle^{-1}}$ will lead to a position $\lambda_{k+1}^{u_{1}}$ such that $\lambda_{k+1}^{u_{1}}$ is associated with a term $\varphi_{\mu(a)}^{3}(b, t, x)$ for which it is the case that

$$
(\forall l)(\forall n)(\forall m)\left[\left(l \leqq k \& n \in \delta \varphi_{u(a)}^{3}(b, m, x) \& m \geqq t\right) \Rightarrow \lambda_{l}<n\right] .
$$

But then, having reached such a stage $u_{1}$, we see from our description of the general stage of the construction that if $\Lambda_{\langle k+1\rangle}{ }^{-1}$ moves after stage $u_{1}$ it does not thereby undergo any further change in the associate of its position. Hence, by the finiteness of the sets in the rows enumerated by $\varphi_{\mu(a)}^{3}$, some finite number of moves of $\left.\Lambda_{\langle k+1\rangle}\right\rangle^{-1}$ subsequent to stage $u_{1}$ will bring it to a position $\lambda_{k+1}^{u_{2}}$ such that $q \geqq u_{2}$ $\Rightarrow \lambda_{k+1}^{q}$ is not insecure (and, hence, $q \geqq u_{2} \Rightarrow \lambda_{k+1}^{q}=\lim _{s \rightarrow \infty} \lambda_{k+1}^{s}$ ). So we see that in fact there cannot be an infinite number of distinct values for $\lambda_{k+1}^{u}$; i.e., $\lim _{s \rightarrow \infty} \lambda_{k+1}^{s}$ exists. By induction on $q$, we thus have that $\lambda_{q}=\lim _{s \rightarrow \infty} \lambda_{q}^{s}$ exists for all $q$.

Next, still under the assumption that $\Sigma[3 ; e]^{F} \subseteq$ Fin, we shall argue that the class $K_{\alpha}=\left\{\left\{x \mid \alpha^{*}(x)\right.\right.$ is defined and $\left.\left.=n\right\} \mid n \in N\right\}$ is a member of $\boldsymbol{F R}$. It is clear from the construction that $\alpha=\bigcup_{s} \alpha^{(s)}$ is a partial recursive function with the unique fixed point 0 ; and it is, moreover, plain from the construction of $\alpha$ that $\rho \alpha \subseteq \delta \alpha$, that $\alpha(n) \leqq n$ for $n \in \delta \alpha$, and that, for each $n,\left\{x \mid \alpha^{*}(x)\right.$ is defined and $\left.=n\right\}=$ the set of all numbers of the form $\lambda_{n}^{s}, s=0,1,2, \ldots$. Since, as was proven above, these latter sets are finite, and since they are plainly nonempty and disjoint by the construction, with $\left\langle\lambda_{n+1}, \lambda_{n}\right\rangle \in \alpha$ for all $n$, we see that $\alpha$ is a finite-to-one special retracing function which in particular retraces the sequence $\lambda_{0}, \lambda_{1}, \lambda_{2}, \ldots$ of final marker positions. So $K_{\alpha} \in \boldsymbol{F R}$.

Now, if $\Sigma[3 ; e]^{F} \nsubseteq$ Fin then we cannot assert that $K_{\alpha} \in \boldsymbol{F R}$; however, $K_{\alpha}$ is in every case an r.e. class of disjoint nonempty sets. Moreover, the construction of $\alpha$ is obviously uniform in the sense that an index of $K_{\alpha}$ is effectively obtainable from $e$; i.e., there is a recursive function $\psi$ such that, for every $e, W_{\psi(e)}^{C}=K_{\alpha}$ where $\alpha$ is the function constructed relative to that particular $e$. As we have just argued, $W_{\psi(e)}^{C}$ is in $\boldsymbol{F R}$ provided $\Sigma[3 ; e]^{F} \subseteq$ Fin; so if we can show that $\Sigma[3 ; e]^{F} \subseteq$ Fin $\Rightarrow W_{\psi(e)}^{C} \neq W_{a}^{C}$ for any $a$ such that $a \in \Sigma[3 ; e]$, then we will have shown that $\psi$ is a $\Sigma_{3}^{0}$-productive function for Fin with $\{$ Fin $; 3 ; \psi\} \subseteq \boldsymbol{F R}$, as required. Supposing, then, that $\Sigma[3 ; e]^{F} \subseteq$ Fin and that $a \in \Sigma[3 ; e] \& W_{\psi(e)}^{C}=W_{a}^{C}$, we see from Lemma B (using the fact that $W_{\psi(e)}^{C} \in \boldsymbol{F R}$ ) that $W_{\psi(e)}^{C}$ is (for some $b$ ) equal to the class of terms enumerated by the $b$ th row, $\varphi_{\mu(a)}(b, z, x)$, of the row-disjoint enumeration

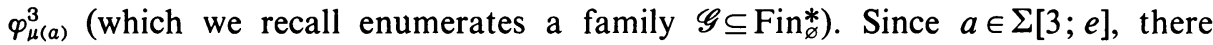
exists a number $c$ such that $(\forall z)(\exists y) T_{3}(e, a, c, z, y)$. Therefore, the marker $\left.\Lambda_{\langle m\rangle}\right\rangle^{-1}$, 
where $\pi_{3}^{-1}(m)=\langle a, b, c\rangle$, can make as many autonomous moves as are required to insure that its final position, $\lambda_{m}$, has the following property:

$$
\begin{aligned}
(\forall l<m)(\forall n)\left[n \in \delta \varphi_{\mu(a)}^{3}(b, t(m), x) \Rightarrow\right. & \left.\lambda_{l}<n\right] \& \\
& (\forall n)\left[n \in \delta \varphi_{\mu(a)}^{3}(b, t(m), x) \Rightarrow n<\lambda_{m}\right],
\end{aligned}
$$

where $\varphi_{\mu(a)}^{3}(b, t(m), x)$ is the term with which $\lambda_{m}$ is associated. (There is no loss of generality in assuming $m \neq 0$.) Since it is plain from the construction that $q>m \Rightarrow \lambda_{q}>\lambda_{m}$, we thus see that the term $\delta \varphi_{\mu(a)}^{3}(b, t(m), x)$ does not contain any number of the form $\lambda_{i}, i=0,1,2, \ldots$ But hence $\delta \varphi_{\mu(a)}^{3}(b, t(m), x)$ is not one of the members of $W_{\psi(e)}^{C}$, and we have a contradiction. This completes the proof of Lemma C.

THEOREM 2. Let $\mathscr{G}$ be any family such that $\boldsymbol{F R} \subseteq \mathscr{G} \subseteq$ Fin. Then $\mathscr{G}$ is $\Sigma_{3}^{0}$-productive and hence, in particular, is not $\Sigma_{3}^{0}$ enumerable.

Proof. Let $\psi$ be as in Lemma C; then the theorem follows at once, since $\{$ Fin; $3 ; \psi\} \subseteq \boldsymbol{F R}$.

We conclude by remarking that the argument used in proving Theorem 2 seems to be tailored rather snugly to the collection of finite sets and to the class of $\Sigma_{3}^{0}$ predicates. We lack at present a general procedure wherewith to attack the levelof-enumerability problem for other families. However, by suitably modifying the techniques of this paper, we are able to show that the family Cofin of all r.e. classes of cofinite subsets of $N$ is $\Sigma_{4}^{0}$-productive. (By an application of our Theorem 1 to a theorem of Mostowski and Rogers, $G$ (Cofin) is $\Pi_{4}^{0}$ complete.) We conjecture, but have no notion how to prove, that the same classification as in the case of Cofin applies to the family of all r.e. classes of recursive sets.

\section{REFERENCES}

1. K. I. Appel and T. G. McLaughlin, On properties of regressive sets, Trans. Amer. Math. Soc. 115 (1965), 83-93. MR 37 \#6176.

2. J. C. E. Dekker, Infinite series of isols, Proc. Sympos. Pure Math., vol. 5, Amer. Math. Soc., Providence, R. I., 1962, pp. 77-96. MR 26 \#16.

3. J. C. E. Dekker and J. R. Myhill, Some theorems on classes of recursively enumerable sets, Trans. Amer. Math. Soc. 89 (1958), 25-59. MR 20 \#3780.

4. S. C. Kleene, Introduction to metamathematics, Van Nostrand, Princeton, N. J., 1952. MR 14, 525.

5. M. B. Pour-El and H. Putnam, Recursively enumerable classes and their application to recursive sequences of formal theories, Arch. Math. Logik Grundlagenforsch. 8 (1965), 104-121. MR 34 \#7370.

6. H. Rogers, Jr., Theory of recursive functions and effective computability, McGraw-Hill, New York, 1967. MR 37 \#61.

7. C. E. M. Yates, Recursively enumerable sets and retracing functions, Z. Math. Logik Grundlagen Math. 8 (1962), 331-345. MR 26 \#3598.

CORNELl UNIVERSITY, ITHACA, New YoRK 14850

UNIVERSITY OF ILLINOIS, URBANA, ILLINOIS 61801 